\title{
Maps on idempotents
}

\author{
by \\ Peter ŠEmRl (Ljubljana)
}

\begin{abstract}
Let $X$ be an infinite-dimensional real or complex Banach space, $B(X)$ the algebra of all bounded linear operators on $X$, and $P(X) \subset B(X)$ the subset of all idempotents. We characterize bijective maps on $P(X)$ preserving commutativity in both directions. This unifies and extends the characterizations of two types of automorphisms of $P(X)$, with respect to the orthogonality relation and with respect to the usual partial order; the latter have been previously characterized by Ovchinnikov. We also describe bijective orthogonality preserving maps on the set of idempotents of a fixed finite rank. As an application we present a nonlinear extension of the structural result for bijective linear biseparating maps on $B(X)$.
\end{abstract}

1. Introduction and statement of main results. Let $X$ be a real or complex Banach space and $B(X)$ the algebra of all bounded linear operators on $X$. Denote by $X^{*}$ the dual of $X$ and by $P(X) \subset B(X)$ the subset of all idempotents. Recall that $P(X)$ is a poset with the partial order defined by $P \leq Q \Leftrightarrow P Q=Q P=P, P, Q \in P(X)$. Orthogonality is another important relation on $P(X)$. Two idempotents $P, Q \in P(X)$ are said to be orthogonal if $P Q=Q P=0$. In this case we write $P \perp Q$. Motivated by some problems in quantum mechanics (see the review MR 95a:46093) Ovchinnikov [16] characterized automorphisms of the poset $P(X)$ in the case that $X$ is a Hilbert space of dimension at least 3. Recall that an automorphism $\phi$ of the poset $P(X)$ is a bijective map preserving order in both directions, that is, $P \leq Q$ if and only if $\phi(P) \leq \phi(Q), P, Q \in P(X)$.

As far as we know, the automorphisms of $P(X)$ with respect to the orthogonality relation have not been treated in the literature. In fact, the structural result for bijective maps on $P(X)$ preserving orthogonality in both directions follows almost directly from the result of Ovchinnikov. Namely, for a subset $\mathcal{S} \subset P(X)$ denote by $\mathcal{S}^{\perp}$ the set of all idempotents from $P(X)$ that are orthogonal to every member of $\mathcal{S}$. In the case $\mathcal{S}=\{P\}$ we simply write $P^{\perp}=\{P\}^{\perp}$. Then, as we shall see, it is easy to verify that for an arbitrary pair of idempotents $P, Q \in P(X)$ we have $P \leq Q$ if and only if

2000 Mathematics Subject Classification: 06A06, 47B49.

This work was supported in part by a grant from the Ministry of Science of Slovenia. 
$Q^{\perp} \subset P^{\perp}$. It follows that every bijective map $\phi: P(X) \rightarrow P(X)$ satisfying $P \perp Q \Leftrightarrow \phi(P) \perp \phi(Q), P, Q \in P(X)$, preserves order in both directions. So, every automorphism of $P(X)$ with respect to $\perp$ is an automorphism with respect to the partial order $\leq$. We will show that also the converse holds true.

Proposition 1.1. Let $X$ be a Banach space and $\phi: P(X) \rightarrow P(X)$ a bijective map. Then $\phi$ is a poset automorphism if and only if $\phi$ preserves orthogonality in both directions, that is, $P \perp Q \Leftrightarrow \phi(P) \perp \phi(Q)$, $P, Q \in P(X)$.

Hence, the problem of characterizing poset automorphisms of $P(X)$ is equivalent to the problem of characterizing orthogonality automorphisms of $P(X)$. So, we can apply the result of Ovchinnikov to get also the characterization of orthogonality automorphisms, or we can solve first the problem of the characterization of orthogonality automorphisms and in this way obtain a new proof of Ovchinnikov's result. We will here solve an even more general problem and then using Proposition 1.1 we will get both results as simple consequences.

A bijective map $\phi: P(X) \rightarrow P(X)$ preserves commutativity in both directions if $P Q=Q P \Leftrightarrow \phi(P) \phi(Q)=\phi(Q) \phi(P), P, Q \in P(X)$. Let $T \in B(X)$ be an invertible operator. Then, clearly, $P \mapsto T P T^{-1}$ is a bijective map on $P(X)$ preserving commutativity in both directions. In the complex case the same is true if $T: X \rightarrow X$ is a bounded invertible conjugate-linear operator. Similarly, if $T: X^{*} \rightarrow X$ is a bounded invertible linear or conjugate-linear operator, then $P \mapsto T P^{*} T^{-1}$ is a bijective map on $P(X)$ preserving commutativity in both directions. Here, $P^{*}$ denotes the adjoint of $P$ (it should be mentioned that the other standard symbol $P^{\prime}$ will be used in this paper to denote the commutant of $P$ ).

Let $\tau: P(X) \rightarrow\{0,1\}$ be a map with the property that $\tau(P)=1$ $\Leftrightarrow \tau(I-P)=1, P \in P(X)$. Define $\xi: P(X) \rightarrow P(X)$ by $\xi(P)=$ $\tau(P) P+(1-\tau(P))(I-P), P \in P(X)$. We will call every such map an ortho-permutation on $P(X)$. For every $P \in P(X)$ it maps either both $P$ and $I-P$ to themselves, or to each other. Clearly, it is a bijective map preserving commutativity in both directions. Our main result states that every bijective map on $P(X)$ preserving commutativity in both directions is a composition of the above-described maps.

THEOREM 1.2. Let $X$ be an infinite-dimensional real Banach space and $\phi: P(X) \rightarrow P(X)$ a bijective map preserving commutativity in both directions. Then there exist an ortho-permutation $\xi: P(X) \rightarrow P(X)$ and either an invertible $T \in B(X)$ such that

$$
\phi(P)=T \xi(P) T^{-1}, \quad P \in P(X),
$$


or a bounded invertible linear operator $T: X^{*} \rightarrow X$ such that

$$
\phi(P)=T \xi(P)^{*} T^{-1}, \quad P \in P(X) .
$$

In the second case $X$ must be reflexive.

THEOREM 1.3. Let $X$ be an infinite-dimensional complex Banach space and $\phi: P(X) \rightarrow P(X)$ a bijective map preserving commutativity in both directions. Then there exist an ortho-permutation $\xi: P(X) \rightarrow P(X)$ and either a bounded invertible linear or conjugate-linear operator $T: X \rightarrow X$ such that

$$
\phi(P)=T \xi(P) T^{-1}, \quad P \in P(X),
$$

or a bounded invertible linear or conjugate-linear operator $T: X^{*} \rightarrow X$ such that

$$
\phi(P)=T \xi(P)^{*} T^{-1}, \quad P \in P(X) .
$$

In the second case $X$ must be reflexive.

As we shall see later it is not difficult to prove that every bijective map on $P(X)$ preserving orthogonality in both directions preserves commutativity in both directions. So, we will easily obtain the following corollaries from our main result and Proposition 1.1.

Corollary 1.4. Let $X$ be an infinite-dimensional real Banach space and $\phi: P(X) \rightarrow P(X)$ a bijective map preserving orthogonality in both directions. Then either there exists an invertible $T \in B(X)$ such that

$$
\phi(P)=T P T^{-1}, \quad P \in P(X),
$$

or there exists a bounded invertible linear operator $T: X^{*} \rightarrow X$ such that

$$
\phi(P)=T P^{*} T^{-1}, \quad P \in P(X) .
$$

In the second case $X$ must be reflexive.

Corollary 1.5. Let $X$ be an infinite-dimensional complex Banach space and $\phi: P(X) \rightarrow P(X)$ a bijective map preserving orthogonality in both directions. Then either there exists a bounded invertible linear or conjugate-linear operator $T: X \rightarrow X$ such that

$$
\phi(P)=T P T^{-1}, \quad P \in P(X),
$$

or there exists a bounded invertible linear or conjugate-linear operator $T$ : $X^{*} \rightarrow X$ such that

$$
\phi(P)=T P^{*} T^{-1}, \quad P \in P(X) .
$$

In the second case $X$ must be reflexive.

Corollary 1.6. Let $X$ be an infinite-dimensional real Banach space and $\phi$ a poset automorphism of $P(X)$. Then either there exists an invertible $T \in B(X)$ such that

$$
\phi(P)=T P T^{-1}, \quad P \in P(X),
$$


or there exists a bounded invertible linear operator $T: X^{*} \rightarrow X$ such that

$$
\phi(P)=T P^{*} T^{-1}, \quad P \in P(X) .
$$

In the second case $X$ must be reflexive.

Corollary 1.7. Let $X$ be an infinite-dimensional complex Banach space and $\phi$ a poset automorphism of $P(X)$. Then either there exists a bounded invertible linear or conjugate-linear operator $T: X \rightarrow X$ such that

$$
\phi(P)=T P T^{-1}, \quad P \in P(X),
$$

or there exists a bounded invertible linear or conjugate-linear operator $T$ : $X^{*} \rightarrow X$ such that

$$
\phi(P)=T P^{*} T^{-1}, \quad P \in P(X) .
$$

In the second case $X$ must be reflexive.

In this paper we will be mainly interested in infinite-dimensional Banach spaces. Let us just mention that in the finite-dimensional complex case the results are slightly more complicated as the map $T$ need not be linear or conjugate-linear, but merely semilinear (see [16]). On the other hand, in the finite-dimensional case the last two corollaries can be proved under weaker assumptions (see [21, 22]).

A lot of attention has been paid to the study of linear maps preserving commutativity on full matrix or operator algebras. Let us mention here just two recent papers covering most of the known results $[4,15]$. The main motivation to study such maps comes from the simple observation that the assumption of preserving commutativity can be considered as the assumption of preserving zero Lie products. The study of nonlinear maps preserving commutativity on full matrix and operator algebras has been initiated recently. In [23] bijective nonlinear maps on full matrix algebras preserving commutativity in both directions were characterized, while in [14] such maps were treated on the Jordan algebra of all self-adjoint operators acting on a Hilbert space. Of course, we will use some of the ideas developed in these two papers. But many of the ideas are essentially different since our situation differs a lot from both the finite-dimensional case and the self-adjoint case.

One of the main motivations for this study was to give a new insight into the beautiful result of Ovchinnikov. His motivation to study automorphisms of the poset of idempotents came from quantum mechanics. Recently, his result was used by Molnár [13] to study another problem in quantum mechanics. Let us briefly describe the background. Wigner's unitary-antiunitary theorem plays a fundamental role in quantum mechanics. It states that every quantum-mechanical invariance transformation can be represented by a unitary or an antiunitary operator on a complex Hilbert space. The reformulation in the mathematical language states that every bijective transfor- 
mation $\phi$ on the set of all one-dimensional linear subspaces of a complex Hilbert space $H$ preserving the angle between every pair of such subspaces (transition probability in the language of quantum mechanics) is induced by a unitary or an antiunitary operator. Uhlhorn [24] improved this result by requiring only that $\phi$ preserves the orthogonality between the one-dimensional subspaces of $H$. This is a significant generalization since Uhlhorn's transformation preserves only the logical structure of the quantum-mechanical system in question while Wigner's transformation preserves its complete probabilistic structure. For some recent results in this direction we refer to $[10,11,13,18]$ where further references can be found.

Recently, Molnár [12] has improved Wigner's theorem in a different way. He characterized bijective transformations on the set of all $n$-dimensional subspaces of a Hilbert space preserving principal angles between such subspaces. In [20] the extension of this result in the spirit of Uhlhorn was proved. We will conclude our discussion on maps on idempotents by treating a nonself-adjoint version of this result. We believe that this kind of result is interesting in itself but some ideas presented in [13] and [19] suggest that it might also be useful when studying the result of Molnár in a more general setting of indefinite inner product spaces.

For a Banach space $X$ and a positive integer $n$ we denote by $P_{n}(X)$ and $P_{\leq n}(X)$ the subset of $P(X)$ consisting of all idempotents of rank $n$ and the subset of $P(X)$ consisting of all idempotents of rank at most $n$, respectively.

TheOREM 1.8. Let $n$ be a positive integer, $X$ an infinite-dimensional real Banach space, and $\phi: P_{n}(X) \rightarrow P_{n}(X)$ a bijective map preserving orthogonality in both directions. Then either there exists an invertible $T \in B(X)$ such that

$$
\phi(P)=T P T^{-1}, \quad P \in P_{n}(X),
$$

or there exists a bounded invertible linear operator $T: X^{*} \rightarrow X$ such that

$$
\phi(P)=T P^{*} T^{-1}, \quad P \in P_{n}(X) .
$$

In the second case $X$ must be reflexive.

Theorem 1.9. Let $n$ be a positive integer, $X$ an infinite-dimensional complex Banach space, and $\phi: P_{n}(X) \rightarrow P_{n}(X)$ a bijective map preserving orthogonality in both directions. Then either there exists a bounded invertible linear or conjugate-linear operator $T: X \rightarrow X$ such that

$$
\phi(P)=T P T^{-1}, \quad P \in P_{n}(X),
$$

or there exists a bounded invertible linear or conjugate-linear operator $T$ : $X^{*} \rightarrow X$ such that

$$
\phi(P)=T P^{*} T^{-1}, \quad P \in P_{n}(X) .
$$

In the second case $X$ must be reflexive. 
The special case when $n=1$ and the underlying field is the field of complex numbers has already been proved in [23]. It was used there to obtain a nonlinear extension of the structural result for Lie automorphisms of the Lie algebra $B(X)$ equipped with the Lie product $[\cdot, \cdot]$ defined by $[A, B]=A B-B A$.

So far we have disscussed mostly the physical motivation for the study of this kind of problems. In the previous paragraph we mentioned an application in the study of Lie automorphisms. But the results of this type are important also in operator theory, for example, when studying the automorphisms of operator semigroups (see [19]). Recently, a lot of work has been done on linear preservers, that is, on linear maps that preserve certain sets or properties. Many problems concerning linear preservers can be reduced to the problem of characterizing linear maps preserving idempotents [8]. In particular, the famous Kaplansky problem on invertibility preserving maps is of this kind $[3,5]$. The restriction of a linear idempotent preserving map $\phi$ on an operator algebra to the poset of all idempotents preserves partial order and orthogonality. Indeed, if $P \leq Q$, then $Q-P$ is an idempotent, and therefore, $\phi(Q)-\phi(P)$ is idempotent, which further implies that $\phi(P) \leq \phi(Q)$. To see that $\phi$ preserves orthogonality one has to observe that the sum of two idempotent operators $P+Q$ is idempotent if and only if $P \perp Q$. So, the structural results obtained in this paper might be helpful when studying linear preservers. Moreover, when reducing a certain linear preserver problem first to the problem of characterizing linear maps preserving idempotents, and then to the problem of characterizing order preserving or orthogonality preserving maps, we ultimately arrive at a nonlinear problem. This may result in a stronger conclusion than we expect at the beginning. We will ilustrate this by considering the problem of characterizing biseparating maps between operator algebras recently studied by Araujo and Jarosz [2].

2. Preliminary results. Let $X$ be a Banach space. For every nonzero $x \in X$ and nonzero $f \in X^{*}$ we denote by $x \otimes f \in B(X)$ the rank one operator defined by $(x \otimes f) z=f(z) x, z \in X$. Note that every rank one operator is of this form. Such an operator is an idempotent if and only if $f(x)=1$. For two rank one idempotents $x \otimes f, y \otimes g \in P(X)$ we write $x \otimes f \sim y \otimes g$ if $x$ and $y$ are linearly dependent or $f$ and $g$ are linearly dependent. In other words, for $P, Q \in P_{1}(X)$ we have $P \sim Q$ if $P$ and $Q$ have the same range space or the same null space.

Lemma 2.1. Let $X$ be a Banach space with $\operatorname{dim} X \geq 3$ and let $P, Q \in$ $P_{1}(X)$. Then $P \sim Q$ if and only if there exist $R, S \in P_{2}(X)$ such that $R \neq S, P \leq R, P \leq S, Q \leq R$, and $Q \leq S$. 
This statement has been known before [16]. As the proof is rather short we give it here for the sake of completeness.

Proof. Assume first that $P \sim Q$. Then $P$ and $Q$ have the same range space or the same null space. We will consider only the first possibility since the proof in the other case is almost the same. So, we have $P=x \otimes f$ and $Q=x \otimes g$ for some $x \in X$ and $f, g \in X^{*}$ with $f(x)=g(x)=1$. There is nothing to prove if $f=g$. So, we may assume that $f \neq g$. Then $f$ and $g$ are linearly independent, and consequently, we can find nonzero $y, z \in X$ such that $f(y)=f(z)=g(z)=0$ and $g(y)=1$. It is now easy to verify that $R=x \otimes f+y \otimes(g-f)$ and $S=x \otimes f+(y+z) \otimes(g-f)$ are distinct rank two idempotents satisfying $P \leq R, P \leq S, Q \leq R$, and $Q \leq S$.

Assume now that $P$ and $Q$ have neither the same range space nor the same null space, and let $R$ be any rank two idempotent with $P, Q \leq R$. Then the range space of $R$ contains both the range spaces of $P$ and $Q$. Thus, the two-dimensional range space of $R$ has to be the direct sum of the range spaces of $P$ and $Q$. Similarly, the null space of $R$, which is of codimension two, must be the intersection of the null spaces of $P$ and $Q$. Every idempotent is completely determined by its range space and its null space. Thus, there exists at most one idempotent $R$ of rank two such that $P, Q \leq R$.

Lemma 2.2. Let $X$ be a Banach space with $\operatorname{dim} X \geq 2$ and let $P, Q \in P_{1}(X)$. Then $P \not \perp Q$ if and only if there exists $R \in P_{1}(X)$ such that $P \sim R$ and $Q \sim R$.

Proof. Let $P=x \otimes f$ and $Q=y \otimes g$. If $P \not \perp Q$, then $f(y) \neq 0$ or $g(x) \neq 0$. In the first case the rank one idempotent $R=\frac{1}{f(y)}(y \otimes f)$ satisfies both conditions $P \sim R$ and $Q \sim R$, while in the second case the choice $R=\frac{1}{g(x)}(x \otimes g)$ gives the idempotent $R$ with the desired properties.

Assume now that $P \perp Q$, that is, $f(y)=g(x)=0$. Suppose also that $R$ is a rank one idempotent satisfying $R \sim P$. Then $R=x \otimes k$ for some $k \in X^{*}$ with $k(x)=1$ or $R=z \otimes f$ for some $z \in X$ with $f(z)=1$. We will treat only the first possibility since the proof in the second case goes through in an almost the same way. Because $P \perp Q$, the vectors $x$ and $y$ are linearly independent, and thus, $Q$ and $R$ have different range spaces. From $k(x)=1$ and $g(x)=0$ we conclude that $k$ and $g$ are linearly independent. Hence, $Q$ and $R$ have different null spaces as well. It follows that $Q \nsim R$, as desired.

For any subset $\mathcal{S} \subset P(X)$ we denote by $\mathcal{S}^{\prime}$ the commutant of $\mathcal{S}$ in $P(X)$, that is, $\mathcal{S}^{\prime}=\{Q \in P(X): Q P=P Q$ for every $P \in \mathcal{S}\}$. In the case $\mathcal{S}=\{P\}$ we simply write $P^{\prime}=\{P\}^{\prime}$. We further denote by $E(X) \subset P(X)$ the subset of all idempotents of rank one or corank one: 
$E(X)=P_{1}(X) \cup\left\{I-P: P \in P_{1}(X)\right\}$. The members of $E(X)$ can be characterized using the commutativity relation in the following way.

Lemma 2.3. Let $X$ be a Banach space with $\operatorname{dim} X \geq 4$ and let $P \in$ $P(X) \backslash\{0, I\}$. Then the following conditions are equivalent:

- $P \in E(X)$,

- for any $Q, P_{1}, Q_{1} \in P(X) \backslash\{0, I\}$ satisfying $P Q=Q P, P^{\prime} \neq Q^{\prime}$, $P_{1} Q_{1}=Q_{1} P_{1}, P_{1}^{\prime} \neq Q_{1}^{\prime}$, and $\{P, Q\}^{\prime} \subset\left\{P_{1}, Q_{1}\right\}^{\prime}$ we have $\{P, Q\}^{\prime}=$ $\left\{P_{1}, Q_{1}\right\}^{\prime}$.

In the case when $\operatorname{dim} X \in\{2,3\}$ every nontrivial idempotent automatically belongs to $E(X)$. The above statement has been inspired by some ideas from [17].

Proof. Assume first that $P \in E(X)$. We have $P^{\prime}=(I-P)^{\prime}$. So, there is no loss of generality in assuming that $P$ is of rank one. Thus, with respect to the direct sum decomposition $X=\operatorname{Im} P \oplus \operatorname{Ker} P$ the operator $P$ has the following matrix representation:

$$
P=\left[\begin{array}{ll}
1 & 0 \\
0 & 0
\end{array}\right]
$$

Assume that $Q, P_{1}, Q_{1} \in P(X) \backslash\{0, I\}$ satisfy $P Q=Q P, P^{\prime} \neq Q^{\prime}$, $P_{1} Q_{1}=Q_{1} P_{1}, P_{1}^{\prime} \neq Q_{1}^{\prime}$, and $\{P, Q\}^{\prime} \subset\left\{P_{1}, Q_{1}\right\}^{\prime}$. From $P Q=Q P$ we conclude that

$$
Q=\left[\begin{array}{cc}
\lambda & 0 \\
0 & R
\end{array}\right]
$$

where $\lambda \in\{0,1\}$ and $R \in P(\operatorname{Ker} P)$. The idempotent $R$ is neither zero nor the identity, since in the first case our assumption $Q \neq 0$ would yield $\lambda=1$, which would further imply that $P^{\prime}=Q^{\prime}$, a contradiction, and the second case is not possible for the same reason. Thus, with respect to the direct sum decomposition $X=\operatorname{Im} P \oplus \operatorname{Im} R \oplus \operatorname{Ker} R$ the idempotents $P$ and $Q$ have the following matrix representations:

$$
P=\left[\begin{array}{lll}
1 & 0 & 0 \\
0 & 0 & 0 \\
0 & 0 & 0
\end{array}\right], \quad Q=\left[\begin{array}{lll}
\lambda & 0 & 0 \\
0 & I & 0 \\
0 & 0 & 0
\end{array}\right] .
$$

It follows that $\{P, Q\}^{\prime}$ consists of all idempotents having the matrix representation of the form

$$
\left[\begin{array}{lll}
\mu & 0 & 0 \\
0 & S & 0 \\
0 & 0 & T
\end{array}\right]
$$

where $\mu \in\{0,1\}$ and $S$ and $T$ are any idempotents. From $\{P, Q\}^{\prime} \subset\left\{P_{1}, Q_{1}\right\}^{\prime}$ we conclude that 


$$
P_{1}=\left[\begin{array}{ccc}
\tau_{1} & 0 & 0 \\
0 & \tau_{2} I & 0 \\
0 & 0 & \tau_{3} I
\end{array}\right], \quad Q_{1}=\left[\begin{array}{ccc}
\tau_{4} & 0 & 0 \\
0 & \tau_{5} I & 0 \\
0 & 0 & \tau_{6} I
\end{array}\right]
$$

with $\tau_{j} \in\{0,1\}, j=1, \ldots, 6$. Replacing $P_{1}$ by $I-P_{1}$, if necessary, we may assume that $\tau_{1}=0$. Similarly, we may and do assume that $\tau_{4}=0$. Now, since $P_{1}$ is a nontrivial idempotent, at least one of $\tau_{2}$ and $\tau_{3}$ is nonzero, and the same holds true for $\tau_{5}$ and $\tau_{6}$. Moreover, we have $\tau_{2} \neq \tau_{5}$ or $\tau_{3} \neq \tau_{6}$, since otherwise we would have $P_{1}=Q_{1}$, a contradiction. We will consider only the case that $\tau_{2} \neq \tau_{5}$ and we will assume with no loss of generality that $\tau_{2}=1$ and $\tau_{5}=0$. It follows that $\tau_{6}=1$. It is now straightforward to conclude that $\{P, Q\}^{\prime}=\left\{P_{1}, Q_{1}\right\}^{\prime}$, as desired.

To prove the other direction assume that $P \notin E(X)$. Then we can find a direct sum decomposition of $X$ into four nontrivial closed subspaces $X=X_{1} \oplus X_{2} \oplus X_{3} \oplus X_{4}$ with $\operatorname{Im} P=X_{1} \oplus X_{2}$ and $\operatorname{Ker} P=X_{3} \oplus X_{4}$. The matrix representation of $P$ with respect to this direct sum decomposition is

$$
P=\left[\begin{array}{llll}
I & 0 & 0 & 0 \\
0 & I & 0 & 0 \\
0 & 0 & 0 & 0 \\
0 & 0 & 0 & 0
\end{array}\right]
$$

Define $Q, P_{1}, Q_{1} \in P(X)$ by

$$
Q=\left[\begin{array}{llll}
I & 0 & 0 & 0 \\
0 & 0 & 0 & 0 \\
0 & 0 & I & 0 \\
0 & 0 & 0 & 0
\end{array}\right], \quad P_{1}=\left[\begin{array}{llll}
I & 0 & 0 & 0 \\
0 & 0 & 0 & 0 \\
0 & 0 & 0 & 0 \\
0 & 0 & 0 & 0
\end{array}\right]
$$

and $Q_{1}=P$. Then, clearly, $P Q=Q P, P^{\prime} \neq Q^{\prime}, P_{1} Q_{1}=Q_{1} P_{1}, P_{1}^{\prime} \neq Q_{1}^{\prime}$, and $\{P, Q\}^{\prime} \subset\left\{P_{1}, Q_{1}\right\}^{\prime}$ but $\{P, Q\}^{\prime} \neq\left\{P_{1}, Q_{1}\right\}^{\prime}$.

Proposition 2.4. Let $X$ be an infinite-dimensional Banach space and $\phi: P_{1}(X) \rightarrow P_{1}(X)$ a bijective map preserving commutativity in both directions. Then either there exists a bounded invertible linear or (in the complex case) conjugate-linear operator $T: X \rightarrow X$ such that

$$
\phi(P)=T P T^{-1}, \quad P \in P_{1}(X),
$$

or there exists a bounded invertible linear or (in the complex case) conjugatelinear operator $T: X^{*} \rightarrow X$ such that

$$
\phi(P)=T P^{*} T^{-1}, \quad P \in P_{1}(X)
$$

In the second case $X$ must be reflexive.

Proof. All we have to do is to show that $\phi$ preserves orthogonality in both directions and then the result in the complex case follows directly from [23, Theorem 2.4]. The real case can be proved in the same way (see a remark following Theorem 2.4 in [23]). 
As $\phi$ and $\phi^{-1}$ satisfy the same assumptions it is enough to show that $\phi$ preserves orthogonality in one direction only. Let $P, Q \in P_{1}(X)$ with $P Q=Q P=0$. In particular, $P$ and $Q$ commute, and therefore, $\phi(P)$ and $\phi(Q)$ is a commuting pair of rank one idempotents. Moreover, by bijectivity we have $\phi(P) \neq \phi(Q)$. It follows that $\phi(P) \perp \phi(Q)$, as desired.

Let $n$ be a positive integer. For $P, Q \in P_{n}(X)$ we write $P \sim Q$ if $\operatorname{rank}(P-Q) \leq 1$. Note that in the case $n=1$ this definition of $\sim$ coincides with the one given at the beginning of this section. We will characterize pairs of idempotents $P, Q \in P_{n}(X)$ satisfying $P \sim Q$. Let $\mathcal{S} \subset P_{n}(X)$. In the rest of this section we will use the symbol $\mathcal{S}^{\perp}$ to denote the orthogonal complement of $\mathcal{S}$ in $P_{n}(X)$, that is, $\mathcal{S}^{\perp}=\left\{R \in P_{n}(X): R S=S R=0\right.$ for every $S \in \mathcal{S}\}$.

Lemma 2.5. Let $n$ be a positive integer and $X$ an infinite-dimensional Banach space. Assume that $P, Q \in P_{n}(X)$ with $P \neq Q$. Then the following statements are equivalent:

- $P \sim Q$,

- $\operatorname{rank}(P-Q)=1$ and either $\operatorname{Im} P=\operatorname{Im} Q$ or $\operatorname{Ker} P=\operatorname{Ker} Q$,

- either there exist a nonzero $x \in \operatorname{Im} P$ and a nonzero $f \in X^{*}$ such that $f(\operatorname{Im} P)=\{0\}$ and $Q=P+x \otimes f$, or there exist a nonzero $x \in \operatorname{Ker} P$ and a nonzero $f \in X^{*}$ such that $f(\operatorname{Ker} P)=\{0\}$ and $Q=P+x \otimes f$,

- for every pair $R, S \in P_{n}(X)$ satisfying $R \neq S$ and $\{P, Q\}^{\perp} \subset\{R, S\}^{\perp}$ we have $\{P, Q\}^{\perp}=\{R, S\}^{\perp}$.

Proof. Assume first that $P \sim Q$. As $P \neq Q$ we have $\operatorname{rank}(P-Q)=1$. Let $P=\sum_{k=1}^{n} z_{k} \otimes g_{k}$, where $z_{1}, \ldots, z_{n} \in X$ are linearly independent and $g_{1}, \ldots, g_{n} \in X^{*}$ are linearly independent. Then $Q=\sum_{k=1}^{n} z_{k} \otimes g_{k}+w \otimes h$ for some nonzero $w \in X$ and some nonzero $h \in X^{*}$. If both sets of vectors $\left\{w, z_{1}, \ldots, z_{n}\right\}$ and $\left\{h, g_{1}, \ldots, g_{n}\right\}$ were linearly independent then $Q$ would be of rank $n+1$, a contradiction. Thus, either $w$ belongs to the linear span of $z_{1}, \ldots, z_{n}$, which yields $\operatorname{Im} Q \subset \operatorname{Im} P$, and because they are of the same rank, we have $\operatorname{Im} Q=\operatorname{Im} P$, or $h$ belongs to the linear span of $g_{1}, \ldots, g_{n}$, which yields $\operatorname{Ker} Q=\operatorname{Ker} P$.

Assume next that $\operatorname{rank}(P-Q)=1$ and either $\operatorname{Im} P=\operatorname{Im} Q$ or $\operatorname{Ker} P=$ $\operatorname{Ker} Q$. Let us consider only the first possibility as the proof in the second case goes through in an almost the same way. So, we have $Q=P+A$ for some rank one operator $A$. From $P z+A z \in \operatorname{Im} P, z \in X$, we deduce that the range space of $A$ is a subspace of the range space of $P$. Thus, there exist $x \in \operatorname{Im} P$ and $f \in X^{*}$ such that $A=x \otimes f$. Since $P+x \otimes f$ is an idempotent of rank $n$ its trace is equal to $n$, and because the trace of $P$ is also $n$, we conclude that $x \otimes f$ is a trace zero operator, and therefore, $f(x)=0$. From $(P+x \otimes f)^{2}=P+x \otimes f$ we get directly $f(\operatorname{Im} P)=\{0\}$. 
To prove the next implication we assume that $Q=P+x \otimes f$ for some nonzero $x \in \operatorname{Im} P$ and some nonzero $f \in X^{*}$ satisfying $f(\operatorname{Im} P)=\{0\}$, and that $R, S \in P_{n}(X)$ satisfy $R \neq S$ and $\{P, Q\}^{\perp} \subset\{R, S\}^{\perp}$. Clearly, $\{P, Q\}^{\perp}=\left\{T \in P_{n}(X): \operatorname{Im} P \subset \operatorname{Ker} T\right.$ and $\operatorname{Im} T \subset$ Ker $P \cap$ Ker $\left.f\right\}$. Assume that $\operatorname{Im} R \not \subset \operatorname{Im} P$. Then we can find $z \in \operatorname{Im} R$ such that $z \notin \operatorname{Im} P$. Thus, $z=z_{1}+z_{2}$ with $z_{1} \in \operatorname{Im} P, z_{2} \in \operatorname{Ker} P$ and $z_{2} \neq 0$. Choose nonzero vectors $y_{1}, \ldots, y_{n} \in \operatorname{Ker} P \cap \operatorname{Ker} f$ such that the set $\left\{z_{2}, y_{1}, \ldots, y_{n}\right\}$ is linearly independent. We can find functionals $f_{1}, \ldots, f_{n} \in X^{*}$ such that $f_{k}(\operatorname{Im} P)=\{0\}$, $k=1, \ldots, n, f_{i}\left(y_{j}\right)=\delta_{i j}, 1 \leq i, j \leq n$, and $f_{1}\left(z_{2}\right)=f_{1}(z) \neq 0$. Here, $\delta_{i j}$ denotes the Kronecker symbol. Then $C=\sum_{k=1}^{n} y_{k} \otimes f_{k} \in\{P, Q\}^{\perp}$ but $C R \neq 0$, a contradiction. It follows that $\operatorname{Im} R \subset \operatorname{Im} P$ and as both these idempotents are of rank $n$, we have $\operatorname{Im} R=\operatorname{Im} P$, and similarly, $\operatorname{Im} S=\operatorname{Im} P$. Further, we can prove in the same way that $\operatorname{Ker} P \cap \operatorname{Ker} f$ $\subset \operatorname{Ker} R$ and Ker $P \cap \operatorname{Ker} f \subset \operatorname{Ker} S$. Now, $R \neq S$ and $\operatorname{Im} R=\operatorname{Im} S$, and consequently, $\operatorname{Ker} R \neq \operatorname{Ker} S$. Thus, $\operatorname{Ker} R \cap \operatorname{Ker} S$ is of codimension at least $n+1$ and because $\operatorname{Ker} P \cap \operatorname{Ker} f \subset \operatorname{Ker} R \cap \operatorname{Ker} S$ we actually have Ker $P \cap$ Ker $f=\operatorname{Ker} R \cap \operatorname{Ker} S$. It follows directly that $\{P, Q\}^{\perp}=\{R, S\}^{\perp}$, as desired. In a similar way we prove that the existence of a nonzero $x \in \operatorname{Ker} P$ and a nonzero $f \in X^{*}$ satisfying $f(\operatorname{Ker} P)=\{0\}$ and $Q=P+x \otimes f$ implies the last condition.

It remains to prove that the last assertion implies the first one. So, let $P$ and $Q$ be a pair of different idempotents of rank $n$ satisfying the last condition. Since $P \neq Q$ we have $\operatorname{Ker} P \neq \operatorname{Ker} Q$ or $\operatorname{Im} P \neq \operatorname{Im} Q$. We will consider only the case that $\operatorname{Ker} P \neq \operatorname{Ker} Q$ since the proof in the other case goes through in almost the same way. In this case the closed subspace $\operatorname{Ker} P \cap \operatorname{Ker} Q$ is a proper subspace of $\operatorname{Ker} P$. Since $Q$ is of finite rank the codimension of Ker $P \cap \operatorname{Ker} Q$ in Ker $P$ is finite. It follows that there exists a finite-dimensional subspace $W \subset X$ such that $\operatorname{Ker} P=(\operatorname{Ker} P \cap \operatorname{Ker} Q) \oplus W$, and consequently, $X=\operatorname{Im} P \oplus(\operatorname{Ker} P \cap \operatorname{Ker} Q) \oplus W$. We choose a nonzero $f \in X^{*}$ satisfying $f(\operatorname{Im} P \oplus(\operatorname{Ker} P \cap \operatorname{Ker} Q))=\{0\}$ and a nonzero $x \in \operatorname{Im} P$. Set $R=P$ and $S=P+x \otimes f$. Obviously, $S$ is an idempotent of rank $n$. We will prove that $\{P, Q\}^{\perp} \subset\{R, S\}^{\perp}$. Indeed, for every $T \in\{P, Q\}^{\perp}$ we have $\operatorname{Im} P \subset \operatorname{Ker} T$, which together with $\operatorname{Im} P=\operatorname{Im} S$ yields $T S=0$. On the other hand, for every such $T$ we have $\operatorname{Im} T \subset \operatorname{Ker} P \cap \operatorname{Ker} Q$, which implies that $f(\operatorname{Im} T)=\{0\}$, and consequently, $S T=0$.

Thus, $\{P, Q\}^{\perp} \subset\{R, S\}^{\perp}$. It follows from our assumption that $\{P, Q\}^{\perp}$ $=\{R, S\}^{\perp}$. Clearly, Ker $P \cap \operatorname{Ker} f$ is a closed subspace of $\operatorname{Ker} P$ of codimension one. Therefore, there exists a nonzero vector $u \in X$ such that $X=\operatorname{Im} P \oplus(\operatorname{Ker} P \cap \operatorname{Ker} f) \oplus \operatorname{span}\{u\}$. Obviously, $\{R, S\}^{\perp}=\left\{T \in P_{n}(X)\right.$ : $T(\operatorname{Im} P)=\{0\}$ and $\operatorname{Im} T \subset \operatorname{Ker} P \cap \operatorname{Ker} f\}$.

We will next prove that $\operatorname{Im} Q=\operatorname{Im} P$. It is enough to show that $\operatorname{Im} Q \subset \operatorname{Im} P$. Assume on the contrary that there exists $z \in \operatorname{Im} Q$ such that 
$z \notin \operatorname{Im} P$. Then $z=z_{1}+z_{2}$ with $z_{1} \in \operatorname{Im} P, z_{2} \in(\operatorname{Ker} P \cap \operatorname{Ker} f) \oplus \operatorname{span}\{u\}$, and $z_{2} \neq 0$. The subspace Ker $P \cap \operatorname{Ker} f$ is infinite-dimensional, and therefore, we can find vectors $w_{1}, \ldots, w_{n} \in \operatorname{Ker} P \cap \operatorname{Ker} f$ such that the set of vectors $\left\{w_{1}, \ldots, w_{n}, z_{2}\right\}$ is linearly independent. Further, we can find functionals $g_{1}, \ldots, g_{n} \in X^{*}$ such that $g_{j}(\operatorname{Im} P)=\{0\}, j=1, \ldots, n, g_{i}\left(w_{j}\right)=\delta_{i j}$, $1 \leq i, j \leq n, g_{1}\left(z_{2}\right)=1$, and $g_{2}\left(z_{2}\right)=\cdots=g_{n}\left(z_{2}\right)=0$. Then, clearly, $T=\sum_{j=1}^{n} w_{j} \otimes g_{j} \in P_{n}(X)$. Moreover, $T \in\{R, S\}^{\perp}$, but $T z=w_{1}$, and consequently, $T Q \neq 0$, a contradiction. Hence, $\operatorname{Im} Q=\operatorname{Im} P$, as desired.

The restriction of $Q$ to the subspace $\operatorname{Ker} P \cap \operatorname{Ker} f$ is the zero operator since otherwise it would be easy to find $T \in\{R, S\}^{\perp}$ with $Q T \neq 0$. So, both $P$ and $Q$ act as the identity on $\operatorname{Im} P=\operatorname{Im} Q$ and they are both zero operators on $\operatorname{Ker} P \cap \operatorname{Ker} f$. Thus, the space $\operatorname{Im} P \oplus(\operatorname{Ker} P \cap \operatorname{Ker} f)$, which has codimension one in $X$, is a subspace of $\operatorname{Ker}(P-Q)$. As $P \neq Q$, we necessarily have $\operatorname{rank}(P-Q)=1$, as desired.

Let $P \in P_{n}(X)$. For every nonzero $x \in \operatorname{Im} P$ we define $L_{P, x}=$ $\left\{P+x \otimes h: h \in X^{*}\right.$ and $\left.h(\operatorname{Im} P)=\{0\}\right\}$. Clearly, $L_{P, x}$ is a subset of $P_{n}(X)$ and $R \sim S$ for every pair $R, S \in L_{P, x}$. Similarly, for every nonzero $y \in \operatorname{Ker} P$ we set $M_{P, y}=\left\{P+y \otimes h: h \in X^{*}\right.$ and Ker $\left.P \subset \operatorname{Ker} h\right\}$. Further, for nonzero $f, g \in X^{*}$ satisfying $f(\operatorname{Im} P)=\{0\}$ and Ker $P \subset$ Ker $g$ we write $R_{P, f}=\{P+u \otimes f: u \in \operatorname{Im} P\}$ and $N_{P, g}=\{P+u \otimes g: u \in$ Ker $P\}$. Each of these sets is contained in $P_{n}(X)$ and if $R, S \in P_{n}(X)$ both belong to one of these subsets then $R \sim S$. We will call these subets of $P_{n}(X)$ basic sets related to $P$. More precisely, for every nonzero $x \in \operatorname{Im} P$ the set $L_{P, x}$ will be called a basic set of type $I$ related to $P$, while for every nonzero $y \in \operatorname{Ker} P$ the set $M_{P, y}$ will be called a basic set of type II related to $P$. Similarly, for nonzero $f, g \in X^{*}$ satisfying $f(\operatorname{Im} P)=\{0\}$ and $\operatorname{Ker} P \subset \operatorname{Ker} g$, the sets $R_{P, f}$ and $N_{P, g}$ will be called basic sets of type III and $I V$, respectively.

Lemma 2.6. Let $n$ be a positive integer and $X$ an infinite-dimensional Banach space. Assume that $\mathcal{S} \subset P_{n}(X)$ is a nonempty subset such that $Q \sim R$ for every pair $Q, R \in \mathcal{S}$. Let $P \in \mathcal{S}$. Then $\mathcal{S}$ is a subset of some basic set related to $P$.

Proof. Define $\mathcal{T}=\mathcal{S}-P=\{Q-P: Q \in \mathcal{S}\}$. Then every member of $\mathcal{T}$ is of rank at most one. Moreover, if $A, B \in \mathcal{T}$, then $\operatorname{rank}(A-B) \leq 1$. Note that the difference of two rank one operators $x \otimes f-y \otimes g$ is of rank at most one if and only if $x$ and $y$ are linearly dependent or $f$ and $g$ are linearly dependent. It follows easily that every subset $\mathcal{C} \subset B(X)$ consisting of operators of rank at most one with the property that $\operatorname{rank}(A-B) \leq 1$, $A, B \in \mathcal{C}$, is contained in $x \otimes X^{*}=\left\{x \otimes f: f \in X^{*}\right\}$ for some nonzero $x \in X$ or in $X \otimes f=\{x \otimes f: x \in X\}$ for some nonzero $f \in X^{*}$. In particular, this holds true for $\mathcal{T}$, and consequently, we have two possibilities, of which we will consider only the first one. So, we asssume from now on 
that there is a nonzero $x \in X$ such that $\mathcal{T} \subset x \otimes X^{*}$. If $\mathcal{S}=\{P\}$ there is nothing to prove. So, we may assume that $\mathcal{S}$ contains at least two different idempotents, or equivalently, there exists a nonzero operator in $\mathcal{T}$. Then by Lemma 2.5, either $x \in \operatorname{Im} P$, or $x \in \operatorname{Ker} P$. Applying Lemma 2.5 once again we easily conclude that in the first case $\mathcal{S} \subset L_{P, x}$, while in the second case $\mathcal{S} \subset M_{P, x}$

Lemma 2.7. Let $n$ be a positive integer, $X$ an infinite-dimensional $B a-$ nach space, and $P \in P_{n}(X)$. Assume that $\mathcal{B} \subset P_{n}(X)$ is a basic set related to $P$. Then $\mathcal{B}^{\perp}=\emptyset$ if and only if $\mathcal{B}$ is of type $I$ or $I V$.

Proof. A straightforward verification is omitted.

Recall that a map $E: U \rightarrow V$ between two real or complex vector spaces is semilinear if it is additive and if there exists an automorphism $\tau$ of the underlying field such that $E(\lambda x)=\tau(\lambda) E x$ for every vector $x \in U$ and every scalar $\lambda$. It is well known that the only automorphism of the real field is the identity. Therefore, every semilinear map between real spaces is automatically linear. Examples of semilinear maps on complex vector spaces are linear and conjugate-linear maps. However, there are many automorphisms of the complex field different from the identity or the complex conjugation [9] and therefore there exist semilinear maps on complex spaces which are neither linear nor conjugate-linear.

Lemma 2.8. Let $n$ be a positive integer, $X$ and $Y$ infinite-dimensional complex Banach spaces, and $E: X \rightarrow Y$ a bijective semilinear map. If $E$ carries closed subspaces of codimension $n$ to closed subspaces of codimension $n$, then $E$ is a linear or conjugate-linear operator.

Proof. The proof of the special case when $n=1$ can be found in [7, Lemma 2]. So, all we have to do is to reduce the general case to this special case, that is, we have to prove that $E$ carries closed hyperplanes to closed hyperplanes. So, let $n>1$ and let $W \subset X$ be a closed hyperplane. Then $W$ can be decomposed into a direct sum $W=W_{1} \oplus W_{2}$ where $W_{1}$ is a closed subspace of $X$ of codimension $n$ and $W_{2}$ is a subspace of dimension $n-1$. We must show that $E W=E W_{1} \oplus E W_{2}$ is a closed subspace. This is true because $E W_{1}$ is closed by our assumption and every direct sum of a closed subspace and a finite-dimensional subspace is closed.

Lemma 2.9. Let $n$ be a positive integer, $X$ and $Y$ infinite-dimensional (both real or both complex) Banach spaces, and $\phi: P_{n}(X) \rightarrow P_{n}(Y)$ a bijective map preserving orthogonality in both directions. Assume further that $E: X^{*} \rightarrow Y$ is a bijective semilinear map such that for every $Q \in P_{n}(X)$ and every $f \in X^{*}$ we have $Q^{*} f=0$ if and only if $\phi(Q) E f=0$. Then $E$ is a bounded invertible linear or (in the complex case) conjugate-linear opera- 
tor and

for every $Q \in P_{n}(X)$.

$$
\phi(Q)=E Q^{*} E^{-1}
$$

Proof. Since $E$ is semilinear there exists an automorphism $\tau$ of the underlying field such that $E(\lambda f)=\tau(\lambda) E f$ for every functional $f \in X^{*}$ and every scalar $\lambda$. Then, clearly, $E^{-1}(\lambda y)=\tau^{-1}(\lambda) E^{-1} y$ for every vector $y \in Y$ and every scalar $\lambda$. It follows that for every $Q \in P_{n}(X)$ the map $E Q^{*} E^{-1}$ : $Y \rightarrow Y$ is linear. Moreover, it is an idempotent of rank $n$. In the next step we will prove that $\operatorname{Ker} \phi(Q)=\operatorname{Ker} E Q^{*} E^{-1}$ and $\operatorname{Im} \phi(Q)=\operatorname{Im} E Q^{*} E^{-1}$. Assume for a moment that this has already been proved. Then, since both $\phi(Q)$ and $E Q^{*} E^{-1}$ are idempotents, they must be equal. In particular, $E Q^{*} E^{-1}$ is bounded for every $Q \in P_{n}(X)$.

By our assumptions, we have $y \in \operatorname{Ker} \phi(Q)$ if and only if $y=E f$ for some $f \in \operatorname{Ker} Q^{*}$, which is equivalent to $y \in \operatorname{Ker} Q^{*} E^{-1}=\operatorname{Ker} E Q^{*} E^{-1}$. Thus, $\operatorname{Ker} \phi(Q)=\operatorname{Ker} E Q^{*} E^{-1}$ for every $Q \in P_{n}(X)$.

Let $W$ be any infinite-dimensional Banach space and $T \in P_{n}(W)$ any idempotent of rank $n$. Then it is easy to verify that $\operatorname{Im} T=\bigcap_{R \in T^{\perp}} \operatorname{Ker} R$ and $\operatorname{Im} T^{*}=\bigcap_{R \in T^{\perp}} \operatorname{Ker} R^{*}$. Now, let $Q$ be any member of $P_{n}(X)$. Then

$$
\begin{aligned}
\operatorname{Im} \phi(Q) & =\bigcap_{R \in \phi(Q)^{\perp}} \operatorname{Ker} R=\bigcap_{R \in Q^{\perp}} \operatorname{Ker} \phi(R) \\
& =\bigcap_{R \in Q^{\perp}} \operatorname{Ker} E R^{*} E^{-1}=\bigcap_{R \in Q^{\perp}} \operatorname{Ker} R^{*} E^{-1}=E\left(\bigcap_{R \in Q^{\perp}} \operatorname{Ker} R^{*}\right) .
\end{aligned}
$$

On the other hand,

$$
\operatorname{Im} E Q^{*} E^{-1}=E\left(\operatorname{Im} Q^{*}\right)=E\left(\bigcap_{R \in Q^{\perp}} \operatorname{Ker} R^{*}\right) .
$$

Thus, $\operatorname{Im} \phi(Q)=\operatorname{Im} E Q^{*} E^{-1}$ for every $Q \in P_{n}(X)$.

Hence, $\phi(Q)=E Q^{*} E^{-1}$ for every $Q \in P_{n}(X)$. It remains to prove that $E$ is linear or conjugate-linear and bounded. In the real case every semilinear map is automatically linear. So, assume in this paragraph that both $X$ and $Y$ are complex spaces. We will show that $E^{-1}$ carries closed subspaces of codimension $n$ to closed subspaces of codimension $n$. Once we prove this we can apply the previous lemma to conclude that $E^{-1}$ is linear or conjugate-linear, and then the same must be true for the mapping $E$. Note that for every closed subspace $U$ of $Y$ of codimension $n$ there exists $Q \in P_{n}(X)$ such that $U=\operatorname{Ker} \phi(Q)$. Now, for every $Q \in P_{n}(X)$ we have $\operatorname{Ker} \phi(Q)=\operatorname{Ker} E Q^{*} E^{-1}=E\left(\operatorname{Ker} Q^{*}\right)$. Thus, $E^{-1}(U)=E^{-1}(\operatorname{Ker} \phi(Q))=$ $\operatorname{Ker} Q^{*}$ is closed.

So, both $E$ and $E^{-1}$ are either linear or conjugate-linear. We will prove that the real linear map $E^{-1}$ has closed graph, which will imply that $E^{-1}$, 
and hence also $E$, is bounded. Assume on the contrary that there exists a sequence $\left(y_{p}\right) \subset Y$ with $y_{p} \rightarrow 0$ and $E^{-1} y_{p} \rightarrow \varphi \neq 0$ as $p \rightarrow \infty$. Find $x_{1} \in X$ with $\varphi\left(x_{1}\right) \neq 0, x_{2}, \ldots, x_{n} \in X$ such that $x_{1}, \ldots, x_{n}$ are linearly independent, and $f_{1}, \ldots, f_{n} \in X^{*}$ such that $f_{i}\left(x_{j}\right)=\delta_{i j}, 1 \leq i, j \leq n$. Then $f_{1}, \ldots, f_{n}$, and hence $E f_{1}, \ldots, E f_{n}$, are linearly independent, and therefore, there exists $m \in Y^{*}$ such that $m\left(E f_{1}\right)=1$ and $m\left(E f_{2}\right)=\cdots=m\left(E f_{n}\right)=0$. Set $Q=\sum_{j=1}^{n} x_{j} \otimes f_{j}$. Then $Q^{*}=\sum_{j=1}^{n} f_{j} \otimes \kappa x_{j}$, where $\kappa: X \rightarrow X^{* *}$ is the canonical embedding. Define the map $\tau$ on the underlying field to be the identity if $E$ is linear, while in the case that $X$ and $Y$ are complex spaces and $E$ is semilinear define $\tau(\lambda)=\bar{\lambda}, \lambda \in \mathbb{C}$. We have

$$
\begin{aligned}
0 & =\lim _{p \rightarrow \infty} m\left(\phi(Q) y_{p}\right)=\lim _{p \rightarrow \infty} m\left(E Q^{*} E^{-1} y_{p}\right) \\
& =\lim _{p \rightarrow \infty} m\left(E\left(\sum_{j=1}^{n} f_{j} \otimes \kappa x_{j}\right) E^{-1} y_{p}\right)=\lim _{p \rightarrow \infty} m\left(E\left(\sum_{j=1}^{n}\left(E^{-1} y_{p}\right)\left(x_{j}\right) f_{j}\right)\right) \\
& =\lim _{p \rightarrow \infty} m\left(\sum_{j=1}^{n} \tau\left(\left(E^{-1} y_{p}\right)\left(x_{j}\right)\right) E f_{j}\right)=\tau\left(\lim _{p \rightarrow \infty}\left(E^{-1} y_{p}\right)\left(x_{1}\right)\right)=\tau\left(\varphi\left(x_{1}\right)\right) \neq 0,
\end{aligned}
$$

a contradiction. This completes the proof.

We will conclude this section by some simple observations concerning bijective orthogonality preserving maps. Let $n$ be a positive integer and $X$ and $Y$ infinite-dimensional real or complex Banach spaces. If $T: X \rightarrow Y$ and $U: X^{*} \rightarrow Y$ are bounded invertible linear or (in the complex case) conjugate-linear maps, then

$$
P \mapsto T P T^{-1} \quad \text { and } \quad P \mapsto U P^{*} U^{-1}
$$

are bijective orthogonality preserving maps from $P_{n}(X)$ onto $P_{n}(Y)$. Let us call them a standard map of type 1 induced by the linear (conjugate-linear) map $T$ and a standard map of type 2 induced by the linear (conjugate-linear) map $U$, respectively.

Our first observation is that each standard map of type 1 differs from every standard map of type 2 . To verify this observe that there exist $P, Q \in$ $P_{n}(X)$ with $P Q=0$ and $Q P \neq 0$. It follows that for each standard map $\phi$ of type 1 we have $\phi(P) \phi(Q)=0$, while for each standard map $\psi$ of type 2 we have $\psi(P) \psi(Q) \neq 0$. Two standard maps of type 1 induced by $T$ and $S$ are equal if and only if $T=\lambda S$ for some nonzero scalar $\lambda$. In this case, of course, either both $T$ and $S$ are linear, or both are conjugate-linear. Indeed, assume that $T P T^{-1}=S P S^{-1}$ for every $P \in P_{n}(X)$. It follows that $T Z=S Z$ for every subspace $Z \subset X$ of dimension $n$. Applying the bijectivity of $T$ and $S$ we see that for every nonzero $x \in X$ we have $T(\operatorname{span}\{x\})=S(\operatorname{span}\{x\})$, or equivalently, $T x=\lambda_{x} S x$ for some scalar $\lambda_{x}$. From

$$
\lambda_{x} S x+\lambda_{y} S y=T x+T y=T(x+y)=\lambda_{x+y} S(x+y)=\lambda_{x+y} S x+\lambda_{x+y} S y
$$


we see that $\lambda_{x}=\lambda_{y}$ whenever $x$ and $y$ are linearly independent. If $x$ and $y$ are linearly dependent then we can find $z$ linearly independent of $x$ and $y$. It follows from the previous step that $\lambda_{x}=\lambda_{z}$ and $\lambda_{y}=\lambda_{z}$. Thus, $\lambda=\lambda_{x}$ is independent of $x$ and $T=\lambda S$. In particular, if $\phi$ and $\psi$ are standard maps of type 1 and if $\phi$ is induced by a linear map and $\psi$ is induced by a conjugatelinear map, then $\phi \neq \psi$. Similarly, two standard maps of type 2 induced by $U$ and $V$ are equal if and only if $U=\lambda V$ for some nonzero scalar $\lambda$.

\section{Proofs of main results}

Proof of Proposition 1.1. Assume first that $\phi$ preserves orthogonality in both directions. Then, as already explained in the introduction, all we have to do is to prove that for an arbitrary pair of idempotents $P, Q \in P(X)$ we have $P \leq Q$ if and only if $Q^{\perp} \subset P^{\perp}$. Assume first that $P \leq Q$. If $R Q=Q R=0$ for some $R \in P(X)$, then $R P=R Q P=0$, and similarly, $P R=0$. Thus, $Q^{\perp} \subset P^{\perp}$.

Suppose now that $Q^{\perp} \subset P^{\perp}$. We have to show that $P \leq Q$, or equivalently, $\operatorname{Im} P \subset \operatorname{Im} Q$ and $\operatorname{Ker} Q \subset \operatorname{Ker} P$. Assume that one of these two conditions, say the first one, is not satisfied. Then we can find a functional $f \in X^{*}$ such that $f(\operatorname{Im} Q)=\{0\}$, but the restriction of $f$ to $\operatorname{Im} P$ is nonzero. Since $f$ is nonzero and $X=\operatorname{Im} Q \oplus \operatorname{Ker} Q$, we can find $x \in \operatorname{Ker} Q$ with $f(x)=1$. It is now easy to verify that $x \otimes f \in Q^{\perp}$, but $x \otimes f \notin P^{\perp}$, a contradiction.

To prove the converse, we assume that $\phi$ is a poset automorphism. First note that for every positive integer $k, \phi$ maps $P_{k}(X)$ onto itself. Indeed, if $P$ is of rank $k$, then we can find a string of idempotents $0=P_{0} \leq P_{1} \leq \cdots \leq$ $P_{k}=P$ with $P_{j} \neq P_{j+1}, j=0, \ldots, k-1$. Thus, $\phi(P)>\phi\left(P_{k-1}\right)>\cdots>0$ is of rank at least $k$. Applying the same argument to the inverse of $\phi$ we see that $\phi$ preserves finite ranks in both directions.

Next observe that two nonzero idempotents $P, Q \in P(X)$ are orthogonal if and only if $R \perp S$ for any rank one idempotents $R, S \in P_{1}(X)$ satisfying $R \leq P$ and $S \leq Q$. The set of all rank one idempotents that are below $P$ is mapped onto the set of all rank one idempotents that are below $\phi(P)$. So, it is enough to show that $\phi$ and $\phi^{-1}$ map every pair of orthogonal rank one idempotents to a pair of the same kind. This follows directly from Lemmas 2.1 and 2.2 .

We will prove the real and the complex case of our main result simultaneously.

Proof of Theorems 1.2 and 1.3. Let $\phi: P(X) \rightarrow P(X)$ be a bijective map preserving commutativity in both directions. Then, clearly, $\phi\left(\mathcal{S}^{\prime}\right)=\phi(\mathcal{S})^{\prime}$ for every subset $\mathcal{S} \subset P(X)$. We have $0^{\prime}=P(X)$ and $I^{\prime}=P(X)$, and for every 
nontrivial idempotent $P$ we have $P^{\prime} \neq P(X)$. Thus, either $\phi(0)=0$ and $\phi(I)=I$, or $\phi(0)=I$ and $\phi(I)=0$. Now, by Lemma 2.3, we have $\phi(E(X))$ $=E(X)$. If $P \in P(X)$ is any idempotent, then there is a unique idempotent $Q \neq P$ satisfying $P^{\prime}=Q^{\prime}$, namely, $Q=I-P$. Therefore, for every $P \in P_{1}(X)$ the pair $\{P, I-P\}$ is mapped to the pair $\{\phi(P), I-\phi(P)\}$. So, after composing $\phi$ with an appropriate ortho-permutation of $P(X)$ which acts as the identity outside $E(X) \cup\{0, I\}$, we may and do assume that $\phi\left(P_{1}(X)\right)=P_{1}(X), \phi(0)=0$, and $\phi(I)=I$. By Proposition 2.4, either there exists a bounded invertible linear or (in the complex case) conjugatelinear operator $T: X \rightarrow X$ such that $\phi(P)=T P T^{-1}, P \in P_{1}(X)$, or there exists a bounded invertible linear or (in the complex case) conjugate-linear operator $T: X^{*} \rightarrow X$ such that $\phi(P)=T P^{*} T^{-1}, P \in P_{1}(X)$. In the second case $X$ is reflexive. Once again we will consider just one of the four cases.

Let us choose the case when $X$ is a complex space and $\phi(P)=T P^{*} T^{-1}$, $P \in P_{1}(X)$, with $T: X^{*} \rightarrow X$ being a conjugate-linear bounded bijective operator. We have to show that for every $P \in P(X)$ we have either $\phi(P)=T P^{*} T^{-1}$ or $\phi(P)=T(I-P)^{*} T^{-1}$. This follows almost directly from the obvious fact that if $P$ and $Q$ are two idempotents such that $\left\{R \in P_{1}(X): R P=P R\right\}=\left\{R \in P_{1}(X): R Q=Q R\right\}$, then either $P=Q$ or $P=I-Q$. Indeed, let $P \in P(X)$ and $\mathcal{S}=\left\{R \in P_{1}(X): R P=P R\right\}$. Then $\left\{R \in P_{1}(X): R \phi(P)=\phi(P) R\right\}=\phi(\mathcal{S})=\left\{T R^{*} T^{-1}: R \in P_{1}(X)\right.$ and $R P=P R\}=\left\{R \in P_{1}(X): R T P^{*} T^{-1}=T P^{*} T^{-1} R\right\}$. Hence, either $\phi(P)=T P^{*} T^{-1}$ or $\phi(P)=T(I-P)^{*} T^{-1}$.

Proof of Corollaries 1.4 and 1.5. Let $\phi: P(X) \rightarrow P(X)$ be a bijective map preserving orthogonality in both directions. We will show that it preserves commutativity in both directions. Clearly, it is enough to see that it preserves commutativity in one direction only, since the inverse of $\phi$ satisfies the same assumptions as $\phi$.

Since 0 is the only idempotent orthogonal to every member of $P(X)$ we have $\phi(0)=0$. Observe first that for every set of pairwise orthogonal idempotents $Q_{1}, \ldots, Q_{k}$ satisfying $Q_{1}+\cdots+Q_{k}=I$, the idempotents $\phi\left(Q_{1}\right), \ldots, \phi\left(Q_{k}\right)$ are also pairwise orthogonal and $\phi\left(Q_{1}\right)+\cdots+\phi\left(Q_{k}\right)$ $=I$. Indeed, if $\phi\left(Q_{1}\right)+\ldots+\phi\left(Q_{k}\right) \neq I$, then there is a nonzero idempotent $R$ that is orthogonal to the idempotent $\phi\left(Q_{1}\right)+\cdots+\phi\left(Q_{k}\right)$, and since $\phi\left(Q_{1}\right), \ldots, \phi\left(Q_{k}\right)$ are pairwise orthogonal we have $R \perp \phi\left(Q_{1}\right), \ldots$, $R \perp \phi\left(Q_{k}\right)$. If $R=\phi(S)$, then $S \perp Q_{1}, \ldots, S \perp Q_{k}$, and consequently, $0 \neq S \perp I$, a contradiction.

Now, let $P_{1}, P_{2} \in P(X)$ commute. Define $Q_{1}=P_{1}\left(I-P_{2}\right), Q_{2}=P_{2}\left(I-P_{1}\right)$, $Q_{3}=P_{1} P_{2}$, and $Q_{4}=I-P_{1}-P_{2}+P_{1} P_{2}$. By the previous paragraph, $\phi\left(Q_{1}\right)+\phi\left(Q_{2}\right)+\phi\left(Q_{3}\right)+\phi\left(Q_{4}\right)=I$. Obviously, $P_{1}, Q_{2}$, and $Q_{4}$ are pairwise orthogonal idempotents whose sum is the identity, and therefore, $\phi\left(P_{1}\right)+$ 
$\phi\left(Q_{2}\right)+\phi\left(Q_{4}\right)=I$, which further yields $\phi\left(P_{1}\right)=\phi\left(Q_{1}\right)+\phi\left(Q_{3}\right)$, and similarly, $\phi\left(P_{2}\right)=\phi\left(Q_{2}\right)+\phi\left(Q_{3}\right)$. Thus, $\phi\left(P_{1}\right)$ and $\phi\left(P_{2}\right)$ commute, as desired.

We can now apply Theorem 1.2 in the real case and Theorem 1.3 in the complex case. In particular, every idempotent of rank one is mapped into $E(X)$. If $P \in P_{1}(X)$, then $\phi(P)$ cannot be of corank one since otherwise $P^{\perp}$ would be an infinite set while $\phi(P)^{\perp}=\{0, I-\phi(P)\}$, a contradiction. Thus, $\phi\left(P_{1}(X)\right)=P_{1}(X)$. So, either there is an invertible bounded linear or (in the complex case) conjugate-linear operator $T: X \rightarrow X$ such that $\phi(P)=T P T^{-1}, P \in P_{1}(X)$, or there exists an invertible bounded linear or (in the complex case) conjugate-linear operator $T: X^{*} \rightarrow X$ such that $\phi(P)=T P^{*} T^{-1}, P \in P_{1}(X)$. In the first case we know that every $Q \in P(X)$ is mapped either to $T Q T^{-1}$ or to $T(I-Q) T^{-1}$. Applying the fact that $\phi(P)=T P T^{-1}, P \in P_{1}(X)$, we easily conclude that the second possibility cannot occur. Similarly, in the second case we get $\phi(Q)=T Q^{*} T^{-1}$ for every $Q \in P(X)$.

Clearly, Corollaries 1.6 and 1.7 follow directly from Proposition 1.1 and Corollaries 1.4 and 1.5.

Proof of Theorems 1.8 and 1.9. Once again we will treat the real and the complex case simultaneously. So, let $X$ be an infinite-dimensional real or complex Banach space, $n$ a positive integer, and $\phi$ a bijective map on $P_{n}(X)$ preserving orthogonality in both directions. Then $\phi\left(\mathcal{S}^{\perp}\right)=(\phi(\mathcal{S}))^{\perp}$ for every $\mathcal{S} \subset P_{n}(X)$. Thus, by Lemma 2.5 we have $P \sim Q$ if and only if $\phi(P) \sim \phi(Q), P, Q \in P_{n}(X)$. Further, let $P \in P_{n}(X)$ be any idempotent of rank $n$. Then, by Lemma 2.6, every basic set related to $P$ is mapped into some basic set related to $\phi(P)$. The inverse of $\phi$ has the same property, and therefore, every basic set related to $P$ is mapped bijectively onto some basic set related to $\phi(P)$. Applying Lemma 2.7 we see that every basic set of type I or IV is mapped bijectively onto some basic set of type I or IV.

Now we are ready for the main step of the proof. We will show that for every $P \in P_{n}(X)$, either there exists a bounded invertible linear or (in the complex case) conjugate-linear operator $T_{P}: X \rightarrow X$ such that $T_{P}(\operatorname{Ker} P)=\operatorname{Ker} \phi(P), T_{P}(\operatorname{Im} P)=\operatorname{Im} \phi(P)$, and

$$
\phi(Q)=T_{P} Q T_{P}^{-1}, \quad Q \in P^{\perp},
$$

or there exists a bounded invertible linear or (in the complex case) conjugatelinear operator $T_{P}: X^{*} \rightarrow X$ such that $T_{P}\left(\operatorname{Ker} P^{*}\right)=\operatorname{Ker} \phi(P), T_{P}\left(\operatorname{Im} P^{*}\right)$ $=\operatorname{Im} \phi(P)$, and

$$
\phi(Q)=T_{P} Q^{*} T_{P}^{-1}, \quad Q \in P^{\perp} .
$$

So, let us fix $P \in P_{n}(X)$. Take a nonzero $x \in \operatorname{Im} P$ and consider $L_{P, x}$, a basic set of type I. We already know that it is mapped onto some basic set of type I or IV. We will consider only the second case because the proof in 
the first case goes through in almost the same way. In fact, the first case is even slightly simpler.

Thus, assume that there is a nonzero $g \in(\operatorname{Im} \phi(P))^{*}$ such that $\phi\left(L_{P, x}\right)=$ $N_{\phi(P), g}$. Hence, for every $h \in X^{*}$ satisfying $h(\operatorname{Im} P)=\{0\}$, that is, for every $h \in \operatorname{Ker} P^{*}$, there exists $u \in \operatorname{Ker} \phi(P)$ such that

$$
\phi(P+x \otimes h)=\phi(P)+u \otimes g .
$$

The map $h \mapsto u$ maps every one-dimensional subspace of Ker $P^{*}$ into some one-dimensional subspace of $\operatorname{Ker} \phi(P)$. Indeed, let $[h]$ be the one-dimensional subspace of Ker $P^{*}$ spanned by a nonzero functional $h$. If $0 \neq k \in \operatorname{Ker} P^{*}$ and $h$ are linearly dependent and $\phi(P+x \otimes h)=\phi(P)+u \otimes g$ and $\phi(P+x \otimes k)$ $=\phi(P)+v \otimes g$, then obviously $\{P, P+x \otimes h\}^{\perp}=\{P, P+x \otimes k\}^{\perp}$, and consequently, $\{\phi(P), \phi(P)+u \otimes g\}^{\perp}=\{\phi(P), \phi(P)+v \otimes g\}^{\perp}$, which further implies that $u$ and $v$ are linearly dependent. Thus, the map $h \mapsto u$ induces a map $\varphi$ from the projective space $\mathbb{P}\left(\operatorname{Ker} P^{*}\right)=\left\{[h]: h \in \operatorname{Ker} P^{*} \backslash\{0\}\right\}$ into $\mathbb{P}(\operatorname{Ker} \phi(P))$. Clearly, $\varphi$ is bijective.

Next we will prove that for every nonzero $f, k, h \in \operatorname{Ker} P^{*}$ we have $[f] \subset[k]+[h]$ if and only if $\varphi([f]) \subset \varphi([k])+\varphi([h])$. There is nothing to prove if $k$ and $h$ are linearly dependent. So, assume they are linearly independent. Let $\phi(P+x \otimes f)=\phi(P)+w \otimes g, \phi(P+x \otimes k)=\phi(P)+z \otimes g$, and $\phi(P+x \otimes h)=\phi(P)+y \otimes g$. Thus, $\varphi([f])=[w], \varphi([k])=[z]$, and $\varphi([h])=[y]$. From $\left\{Q \in P_{n}(X): Q(\operatorname{Im} P)=\{0\}\right.$ and $\left.\operatorname{Im} Q \subset \operatorname{Ker} P \cap \operatorname{Ker} k \cap \operatorname{Ker} h\right\}$ $=\{P, P+x \otimes k, P+x \otimes h\}^{\perp} \subset\{P, P+x \otimes f\}^{\perp}$ we get $\{\phi(P)$, $\phi(P)+z \otimes g, \phi(P)+y \otimes g\}^{\perp} \subset\{\phi(P), \phi(P)+w \otimes g\}^{\perp}$, which yields the desired inclusion $[w] \subset[z]+[y]$. In the same way we prove that $\varphi([f]) \subset$ $\varphi([k])+\varphi([h])$ implies $[f] \subset[k]+[h]$. By the fundamental theorem of projective geometry there exists a bijective semilinear map $E: \operatorname{Ker} P^{*} \rightarrow \operatorname{Ker} \phi(P)$ such that $\varphi([f])=[E f], f \in \operatorname{Ker} P^{*} \backslash\{0\}$. In other words, for every $f \in \operatorname{Ker} P^{*} \backslash\{0\}$ there exists a nonzero scalar $\mu$ such that $\phi(P+x \otimes f)=$ $\phi(P)+\mu E f \otimes g$.

Now, $P^{\perp}$ can be identified with $P_{n}(\operatorname{Ker} P)$ because every $Q \in P_{n}(\operatorname{Ker} P)$ can be extended to $Q: X \rightarrow X=\operatorname{Ker} P \oplus \operatorname{Im} P$ with the extension acting as the zero operator on $\operatorname{Im} P$. Clearly, such an extension belongs to $P^{\perp}$. Similarly, $\phi(P)^{\perp}$ can be identified with $P_{n}(\operatorname{Ker} \phi(P))$. We consider the restriction of $\phi$ to $P^{\perp}$ as a bijective orthogonality preserving map from $P^{\perp}$ onto $\phi(P)^{\perp}$. Also, Ker $P^{*} \subset X^{*}$ can be identified in the natural way with $(\operatorname{Ker} P)^{*}$. So, in order to see that we can apply Lemma 2.9 to the restriction $\phi: P^{\perp} \rightarrow \phi(P)^{\perp}$ we have to show that the map $E$ defined in the previous paragraph satisfies the assumption of that lemma. To see this assume that $Q \in P^{\perp}$ and $f \in \operatorname{Ker} P^{*}$. Then $Q^{*} f=0$ if and only if $Q \perp P+x \otimes f$, which is equivalent to $\phi(Q) \perp \phi(P)+\mu E f \otimes g$, which holds true if and only if $\phi(Q) E f=0$. 
By Lemma 2.9, the map $E: \operatorname{Ker} P^{*} \rightarrow \operatorname{Ker} \phi(P)$ is a bounded invertible linear or conjugate-linear map. We can extend $E$ to a bijective bounded linear or conjugate-linear map from $X^{*}$ onto $X$, such that this extension maps $\operatorname{Im} P^{*}$ bijectively onto $\operatorname{Im} \phi(P)$. Denoting this extension by $T_{P}$ we complete the main step of the proof.

Hence, now we know that for every $P \in P_{n}(X)$, either there exists a bounded invertible linear or (in the complex case) conjugate-linear operator $T_{P}: X \rightarrow X$ such that $T_{P}(\operatorname{Ker} P)=\operatorname{Ker} \phi(P), T_{P}(\operatorname{Im} P)=\operatorname{Im} \phi(P)$, and

$$
\phi(Q)=T_{P} Q T_{P}^{-1}, \quad Q \in P^{\perp},
$$

or there exists a bounded invertible linear or (in the complex case) conjugatelinear operator $T_{P}: X^{*} \rightarrow X$ such that $T_{P}\left(\operatorname{Ker} P^{*}\right)=\operatorname{Ker} \phi(P), T_{P}\left(\operatorname{Im} P^{*}\right)$ $=\operatorname{Im} \phi(P)$, and

$$
\phi(Q)=T_{P} Q^{*} T_{P}^{-1}, \quad Q \in P^{\perp} .
$$

Using the terminology from the end of the previous section we can reformulate the above statement by saying that for every $P \in P_{n}(X)$ the restriction $\phi_{\mid P^{\perp}}: P^{\perp} \rightarrow \phi(P)^{\perp}$ is a standard map of type 1 or 2 induced by a linear or a conjugate-linear map. So, for each $P$ we have two possibilities in the real case and four possibilities in the complex case. Let $P$ and $Q$ be any two members of $P_{n}(X)$ with $P \perp Q$. Considering the restriction of $\phi$ to $\{P, Q\}^{\perp}$ and applying the remarks from the end of the previous section we conclude that the restrictions of $\phi$ to $P^{\perp}$ and $Q^{\perp}$ must be of the same type.

Once again we have several cases to treat and as they are similar we will consider only one of them. So, assume that $X$ is a complex space and that for every $P \in P_{n}(X)$ there exists a bounded invertible conjugate-linear operator $T_{P}: X^{*} \rightarrow X$ such that $T_{P}\left(\operatorname{Ker} P^{*}\right)=\operatorname{Ker} \phi(P), T_{P}\left(\operatorname{Im} P^{*}\right)=\operatorname{Im} \phi(P)$, and

$$
\phi(Q)=T_{P} Q^{*} T_{P}^{-1}, \quad Q \in P^{\perp} .
$$

As at the end of the previous section, we conclude that the restriction of $T_{P}$ to the subspace $\operatorname{Ker} P^{*} \cap \operatorname{Ker} Q^{*}$ is a scalar multiple of the restriction of $T_{Q}$ to this subspace whenever $P \perp Q, P, Q \in P_{n}(X)$. Now, let $P, Q \in P_{n}(X)$ be arbitrary idempotents of rank $n$. By the previous step we know that for every $R \in P_{n}(X)$ with $P \perp R$ and $Q \perp R$ the restrictions of $T_{P}$ and $T_{Q}$ to $\operatorname{Ker} P^{*} \cap \operatorname{Ker} Q^{*} \cap \operatorname{Ker} R^{*}$ are the same up to a multiplicative constant. Here, $R$ was any idempotent orthogonal to both $P$ and $Q$. It follows that the restriction of $T_{P}$ to the subspace $\operatorname{Ker} P^{*} \cap \operatorname{Ker} Q^{*}$ is a scalar multiple of the restriction of $T_{Q}$ to this subspace in this general case as well. By absorbing a constant we may assume that for every pair $P, Q \in P_{n}(X)$ we have

$$
T_{P} f=T_{Q} f
$$

for every $f \in \operatorname{Ker} P^{*} \cap \operatorname{Ker} Q^{*}$. So, the map $T: X^{*} \rightarrow X$ given by $T f=T_{P} f$, $f \in \operatorname{Ker} P^{*}$ is well defined. Clearly, this is a bounded invertible conjugate- 
linear map. It is now easy to verify that $\phi(Q)=T Q^{*} T^{-1}$ for every $Q \in$ $P_{n}(X)$.

4. An application. Let $X$ be an infinite-dimensional Banach space. A bijective map $\phi: B(X) \rightarrow B(X)$ is called biseparating if $A B=0 \Leftrightarrow$ $\phi(A) \phi(B)=0, A, B \in B(X)$. Recently, Araujo and Jarosz [2] proved that every linear bijective biseparating map on $B(X)$ is a scalar multiple of an inner automorphism of $B(X)$. An interested reader can find more information on separating and biseparating linear maps in [1] and [6].

Applying our results we can study the more general problem of characterizing nonlinear biseparating maps on $B(X)$. Clearly, every bijective map $\eta: B(X) \rightarrow B(X)$ with the property that $\overline{\operatorname{Im} \eta(A)}=\operatorname{Im} A$ and $\operatorname{Ker} \eta(A)=\operatorname{Ker} A, A \in B(X)$, is biseparating. Every such map will be called a bijective kernel-image preserving map. We will prove that every bijective biseparating map on $B(X)$ is a composition of such a map and an inner linear or (in the complex case) conjugate-linear automorphism of $B(X)$. The result of Araujo and Jarosz is a rather easy consequence.

THEOREM 4.1. Let $X$ be an infinite-dimensional Banach space and $\phi$ : $B(X) \rightarrow B(X)$ a bijective biseparating map. Then there exist a bijective kernel-image preseving map $\eta: B(X) \rightarrow B(X)$ and a bounded bijective linear or (in the complex case) conjugate-linear map $T: X \rightarrow X$ such that $\phi(A)=\operatorname{T\eta }(A) T^{-1}, A \in B(X)$.

Proof. Clearly, $\phi(0)=0$. Denote by $B_{1}(X) \subset B(X)$ the subset of all rank one operators. For $A \in B(X)$ we define $A^{\perp}=\{B \in B(X): B A=A B=0\}$ and $A^{\perp \perp}=\left\{B \in B(X): B C=C B=0\right.$ for every $\left.C \in A^{\perp}\right\}$. We have $\phi\left(A^{\perp}\right)=\phi(A)^{\perp}$ and $\phi\left(A^{\perp \perp}\right)=\phi(A)^{\perp \perp}$ for every $A \in B(X)$.

Let us prove that for a nonzero $A \in B(X)$ the following statements are equivalent:

- $A \in B_{1}(X)$,

- for every nonzero $B \in A^{\perp \perp}$ we have $B^{\perp}=A^{\perp}$.

Assume for a moment that this equivalence has already been proved. Then, clearly, $\phi\left(B_{1}(X)\right)=B_{1}(X)$.

In order to prove the above equivalence we first assume that $A=x \otimes f$ is a rank one operator and $B \in B(X)$ is a nonzero operator belonging to $A^{\perp \perp}$. Then $A^{\perp}=\{C \in B(X): C x=0$ and $\operatorname{Im} C \subset \operatorname{Ker} f\}$. We will prove that $\operatorname{Im} B \subset \operatorname{span}\{x\}$ and $\operatorname{Ker} f \subset \operatorname{Ker} B$. Let us prove just one of these two inclusions, say, the second one. Assume on the contrary that there is a nonzero $z \in X$ such that $z \in \operatorname{Ker} f$ but $z \notin \operatorname{Ker} B$. Take any nonzero $g \in X^{*}$ satisfying $g(x)=0$. Then, obviously, $z \otimes g \in A^{\perp}$, which yields $B \cdot z \otimes g=(B z) \otimes g=0$, a contradiction. 
Now, since $B$ is nonzero we have $\operatorname{Im} B=\operatorname{span}\{x\}$ and $\operatorname{Ker} f=\operatorname{Ker} B$. It follows that $B=\lambda A$ for some nonzero scalar $\lambda$, and consequently, $B^{\perp}=A^{\perp}$, as desired.

To prove the converse suppose that $A \in B(X)$ is an operator whose range space is at least two-dimensional. Take any nonzero $w \in \operatorname{Im} A$ and any nonzero $k \in X^{*}$ satisfying $k(\operatorname{Ker} A)=\{0\}$. If $A C=C A=0$, then $C(\operatorname{Im} A)=\{0\}$ and $\operatorname{Im} C \subset \operatorname{Ker} A$, which further implies that $C B=B C=0$, where $B=w \otimes k$. Thus, $B \in A^{\perp \perp}$. Since $\operatorname{Im} A$ is at least two-dimensional we can find $g \in X^{*}$ with $g(w)=0$ and $g(\operatorname{Im} A) \neq\{0\}$. Choose a nonzero $u \in \operatorname{Ker} k$. Then $u \otimes g \in B^{\perp}$ but $u \otimes g \notin A^{\perp}$. This completes the proof of the above equivalence.

In the next step we will prove that for every $P \in P_{1}(X)$ there exist a nonzero scalar $\lambda_{P}$ and $Q \in P_{1}(X)$ such that $\phi(P)=\lambda_{P} Q$. Every rank one operator is either a scalar multiple of a rank one idempotent, or a square-zero operator. So, all we have to do is to show that for every $P \in P_{1}(X)$ the rank one operator $\phi(P)$ is not square-zero. If $\phi(P)^{2}=0$, then we would have $P^{2}=0$, a contradiction.

Hence, for every $P \in P_{1}(X)$ there exist a nonzero scalar $\lambda_{P}$ and $Q \in P_{1}(X)$ such that $\phi(P)=\lambda_{P} Q$. The scalar $\lambda_{P}$ and the rank one idempotent $Q$ are uniquely determined. Define a map $\varphi: P_{1}(X) \rightarrow P_{1}(X)$ by $\varphi(P)=Q$. Obviously, this is a bijective map preserving orthogonality in both directions. By Theorems 1.8 and 1.9 with $n=1$, either there exists a bounded invertible linear or (in the complex case) conjugate-linear operator $T: X \rightarrow X$ such that $\varphi(P)=T P T^{-1}, P \in P_{1}(X)$, or there exists a bounded invertible linear or (in the complex case) conjugate-linear operator $T: X^{*} \rightarrow X$ such that $\varphi(P)=T P^{*} T^{-1}, P \in P_{1}(X)$. It is easy to find rank one idempotents $P$ and $Q$ such that $P Q=0$ but $Q P \neq 0$, and consequently, $P^{*} Q^{*} \neq 0$. Thus, the second possibility cannot occur. After composing $\phi$ with the similarity transformation $A \mapsto T^{-1} A T$ we may assume with no loss of generality that for every $P \in P_{1}(X)$ there exists a nonzero scalar $\lambda_{P}$ such that $\phi(P)=\lambda_{P} P$.

Let $A \in B(X)$ be any operator. If $\operatorname{Ker} A \not \subset \operatorname{Ker} \phi(A)$, then we can easily find $P \in P_{1}(X)$ such that $A P=0$ but $\phi(A) P \neq 0$. It follows that $\phi(A) \phi(P)=\lambda_{P} \phi(A) P=0$, a contradiction. So, $\operatorname{Ker} A \subset$ $\operatorname{Ker} \phi(A)$ and we prove similarly that $\operatorname{Ker} \phi(A) \subset \operatorname{Ker} A$ and $\overline{\operatorname{Im} A}=$ $\overline{\operatorname{Im} \phi(A)}$.

Corollary 4.2. Let $X$ be an infinite-dimensional Banach space and $\phi: B(X) \rightarrow B(X)$ a bijective additive biseparating map. Then there exist a nonzero scalar $\lambda$ and a bounded bijective linear or (in the complex case) conjugate-linear map $T: X \rightarrow X$ such that $\phi(A)=\lambda T A T^{-1}$, $A \in B(X)$. 
Proof. All we have to do is to prove that every additive kernel-image preserving map $\eta: B(X) \rightarrow B(X)$ is of the form $\eta(A)=\lambda A, A \in B(X)$, for some nonzero scalar $\lambda$. Clearly, for every $R \in B_{1}(X)$ there exists a nonzero scalar $\lambda_{R}$ such that $\eta(R)=\lambda_{R} R$.

We will first prove that $\lambda_{R}=\lambda$ is independent of the choice of $R \in B_{1}(X)$. Let $x, y \in X$ be any linearly independent vectors and $f, g \in X^{*}$ any linearly independent linear functionals. From the fact that the null space of $\phi(x \otimes(f+g))=\phi(x \otimes f)+\phi(x \otimes g)=x \otimes\left(\lambda_{x \otimes f} f+\lambda_{x \otimes g} g\right)$ is the null space of the functional $f+g$ we conclude that $\lambda_{x \otimes f}=\lambda_{x \otimes g}$, and similarly, $\lambda_{x \otimes g}=\lambda_{y \otimes g}$. It follows that $\lambda_{x \otimes f}=\lambda_{y \otimes g}$.

If $x$ and $y$ are linearly dependent or $f$ and $g$ are linearly dependent, then we can find $z \in X$ and $k \in X^{*}$ such that all pairs $x, z$, and $y, z$, and $f, k$, and $g, k$ are linearly independent and then by the previous step we have $\lambda_{x \otimes f}=\lambda_{z \otimes k}=\lambda_{y \otimes g}$. Thus, $\lambda_{R}$ is independent of $R \in B_{1}(X)$.

Let $A \in B(X)$ be any operator. We have to prove that $\eta(A)=\lambda A$. Choose any $x \in X$ and define $A x=y$. Find a functional $k \in X^{*}$ satisfying $k(x)=1$. Then $x \in \operatorname{Ker}(A-y \otimes k)$, and consequently, $x \in \operatorname{Ker} \phi(A-y \otimes k)=$ $\operatorname{Ker}(\phi(A)-\lambda y \otimes k)$, which yields $\phi(A) x=\lambda y$, as desired.

It should be mentioned that the result of Araujo and Jarosz covers also the finite-dimensional case as well as some other operator algebras. On the other hand, they have considered linear maps, while we have here a weaker assumption of additivity. Slightly modifying our methods we can extend this additive version to more general standard unital operator algebras. Our main motivation to include this result was to indicate how our main results can be applied to obtain nonlinear extensions of some linear preserver results, and it is easier to present this idea if one does not need to take care of technicalities necessary for more general results that can be obtained using essentially the same approach.

Acknowledgements. I would like to thank N.-C. Wong for a fruitful discussion on separating and biseparating maps.

\section{References}

[1] Y. A. Abramovich and A. K. Kitover, Inverses of disjointness preserving operators, Mem. Amer. Math. Soc. 143 (2000), no. 679.

[2] J. Araujo and K. Jarosz, Biseparating maps between operator algebras, J. Math. Anal. Appl. 282 (2003), 48-55.

[3] B. Aupetit, Spectrum-preserving linear mappings between Banach algebras or Jordan-Banach algebras, J. London Math. Soc. (2) 62 (2000), 917-924.

[4] M. Brešar, Commuting traces of biadditive mappings, commutativity preserving mappings, and Lie mappings, Trans. Amer. Math. Soc. 335 (1993), 525-546. 
[5] M. Brešar and P. Šemrl, Spectral characterization of idempotents and invertibility preserving linear maps, Exposition. Math. 17 (1999), 185-192.

[6] M. A. Chebotar, W.-F. Ke, P.-H. Lee, and N.-C. Wong, Mappings preserving zero products, Studia Math. 155 (2003), 77-94.

[7] P. A. Fillmore and W. E. Longstaff, On isomorphisms of lattices of closed subspaces, Canad. J. Math. 36 (1984), 820-829.

[8] A. Guterman, C.-K. Li, and P. Šemrl, Some general techniques on linear preserver problems, Linear Algebra Appl. 315 (2000), 61-81.

[9] H. Kestelman, Automorphisms of the field of complex numbers, Proc. London Math. Soc. (2) 53 (1951), 1-12.

[10] L. Molnár, An algebraic approach to Wigner's unitary-antiunitary theorem, J. Austral. Math. Soc. Ser. A 65 (1998), 354-369.

[11] - Generalization of Wigner's unitary-antiunitary theorem for indefinite inner product spaces, Comm. Math. Phys. 210 (2000), 785-791.

[12] - , Transformations on the set of all $n$-dimensional subspaces of a Hilbert space preserving principal angles, ibid. 217 (2001), 409-421.

[13] - Orthogonality preserving transformations on indefinite inner product spaces: generalization of Uhlhorn's version of Wigner's theorem, J. Funct. Anal. 194 (2002), 248-262.

[14] L. Molnár and P. Šemrl, Non-linear commutativity preserving maps on self-adjoint operators, preprint.

[15] M. Omladič, H. Radjavi, and P. Šemrl, Preserving commutativity, J. Pure Appl. Algebra 156 (2001), 309-328.

[16] P. G. Ovchinnikov, Automorphisms of the poset of skew projections, J. Funct. Anal. 115 (1993), 184-189.

[17] C. E. Rickart, Isomorphic groups of linear transformations, Amer. J. Math. 72 (1950), 451-464.

[18] P. Šemrl, Generalized symmetry transformations on quaternionic indefinite inner product spaces: An extension of quaternionic version of Wigner's theorem, Comm. Math. Phys. 242 (2003), 579-584.

[19] - Applying projective geometry to transformations on rank one idempotents, J. Funct. Anal. 210 (2004), 248-257.

[20] - Orthogonality preserving transformations on the set of $n$-dimensional subspaces of a Hilbert space, Illinois J. Math. 48 (2004), 567-573.

[21] - Order-preserving maps on the poset of idempotent matrices, Acta Sci. Math. (Szeged) 69 (2003), 481-490.

[22] - , Hua's fundamental theorem of the geometry of matrices, J. Algebra 272 (2004), 801-837.

[23] - Non-linear commutativity preserving maps, preprint.

[24] U. Uhlhorn, Representation of symmetry transformations in quantum mechanics, Ark. Fys. 23 (1963), 307-340.

Department of Mathematics

University of Ljubljana

Jadranska 19

SI-1000 Ljubljana, Slovenia

E-mail: peter.semrl@fmf.uni-lj.si 